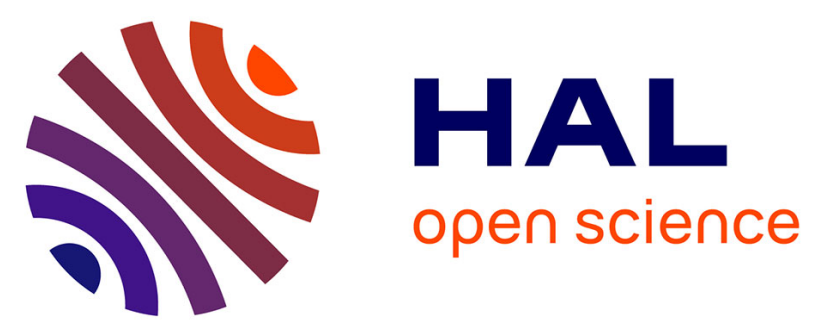

\title{
AMORPHOUS FILMS OF TRANSITION AND COINAGE METALS : SOME STRUCTURAL, ELECTRICAL AND MAGNETIC PROPERTIES
}

L. Davies, P. Grundy, S. Nandra

\section{- To cite this version:}

L. Davies, P. Grundy, S. Nandra. AMORPHOUS FILMS OF TRANSITION AND COINAGE METALS: SOME STRUCTURAL, ELECTRICAL AND MAGNETIC PROPERTIES. Journal de Physique Colloques, 1975, 36 (C2), pp.C2-59-C2-64. 10.1051/jphyscol:1975211 . jpa-00216258

\section{HAL Id: jpa-00216258 https://hal.science/jpa-00216258}

Submitted on 1 Jan 1975

HAL is a multi-disciplinary open access archive for the deposit and dissemination of scientific research documents, whether they are published or not. The documents may come from teaching and research institutions in France or abroad, or from public or private research centers.
L'archive ouverte pluridisciplinaire HAL, est destinée au dépôt et à la diffusion de documents scientifiques de niveau recherche, publiés ou non, émanant des établissements d'enseignement et de recherche français ou étrangers, des laboratoires publics ou privés. 


\title{
AMORPHOUS FILMS OF TRANSITION AND COINAGE METALS : SOME STRUCTURAL, ELECTRICAL AND MAGNETIC PROPERTIES
}

\author{
L. B. DAVIES $(*)$, P. J. GRUNDY and S. S. NANDRA
}

Department of Pure and Applied Physics

University of Salford, Salford M5 4WT, U. K.

\begin{abstract}
Résumé. - On fait état de résultats expérimentaux concernant la structure de films monoatomiques amorphes de métaux de transition et de métaux nobles obtenus par évaporation sous vide.

Ces résultats sont exploités à l'aide de modèles structuraux proposés récemment. Après avoir rappelé les méthodes de détermination de structure par diffraction électronique par transmission qu'on a utilisées, on effectue une revue critique des dernières techniques expérimentales d'investigation. La cristallisation de ces films métastables a été suivie par diffraction électronique et mesure de la résistance électrique, et on présente les premiers résultats de mesures magnétiques obtenus sur les films ferromagnétiques.
\end{abstract}

\begin{abstract}
This paper considers experimental information on the atomic arrangements in non-crystalline single element films of transition and coinage metals prepared by vapour quenching and compares this information with results from recently reported structural models. The method of structural analysis using transmission electron diffraction is outlined and several recent experimental investigations are discussed. The crystallization of the metastable films is followed by electron scattering and electrical resistance measurements and some preliminary magnetic measurements on the ferromagnetic films are described.
\end{abstract}

1. Introduction. - In recent years there have been many studies made of the structural and physical properties of amorphous metallic films, both alloy films [1-4] and films of a single element [5-9]. These investigations include detailed structural investigations [2, 4, 7 and 9] electrical resistance measurements $[8,10]$ and magnetic measurements $[1,6]$. In the main attention has been focused on films of transition metals, either alloyed with a glass forming element or in the pure form. Measurements have also been reported on amorphous coinage metal films [10]. There are several methods available for preparing thin and thick films of amorphous metals, e. g. splat cooling [1,4], chemical [3] and electrochemical deposition [2] and vapour quenching [5-10]. Single element metal films of the first transition series and coinage metal group are usually metastable at room temperature, having no glass former in the accepted sense to stabilise them, and have been prepared by vapour quenching i. e. deposition onto a cold substrate. In this paper we present somewhat of a review of our own previously determined atomic arrangement information for these films and compare it with other recent investigations. We consider the agreement between these experimental results and recent calculations of atomic arrangements $[9,11-14]$ and scattering $[9,13-14]$ in models

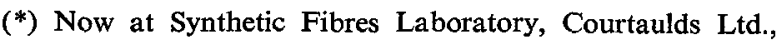
Coventry. of random packing. We consider some electrical resistance measurements and some preliminary magnetic measurements on the metastable films during crystallization. The non-crystalline films discussed in this paper are termed amorphous as it has become clear, as will be shown, that the atomic arrangements in them and in particular the electron scattering from them cannot be associated with microcrystalline structures so far considered.

2. Film preparation and experimental methods. All the films described in this paper were prepared by vacuum deposition onto substrates cooled by liquid nitrogen or helium. In the case of transmission electron diffraction experiments the substrates were either thin collodion, carbon or formvar films [7, 9, 14] and for the electrical resistance [8, 10], magneto electric [15] and magnetic measurements the substrates were sapphire or quartz wafers. Our depositions were made on liquid nitrogen cooled substrates in a differentially pumped electron microscope in vacua of about $10^{-5}$. $10^{-6}$ torr. Ichikawa [9], Bennett and Wright [8] and Leung and Wright [14] used liquid helium cooled substrates in specially constructed electron diffraction systems, the first at $10^{-6}$ torr and the last two investigations in UHV conditions of $10^{-8}-10^{-9}$ torr. The present authors, and Ichikawa, recorded the electron scattering patterns on photographic plates, and direct recording using a scanning technique with 
velocity filtering has been used by Wright and his coworkers. The electrical resistance measurements were made by conventional d. c. methods $[8,10]$ and the magnetization of the ferromagnetic films were obtained in a sensitive Faraday balance modified for insitu deposition of thin films. The details of this apparatus will be given elsewhere [16].

3. Structural analysis. - In our studies the structure sensitive intensity or interference function, $I_{\mathrm{m}}(s)$, was extracted from the total scattering intensity $I(s)$ by removing contributions due to the atomic scattering of the specimen, the elastic scattering from the substrate and inelastic scattering from the specimen and substrate. This is a standard procedure, see e. g. $[5,9,17]$, for scattering patterns recorded photographically. In direct recording scanning techniques the procedure is much simplified, especially with velocity filtering for removing inelastic electrons, and the measurements are probably less subject to error. Multiple scattering was ignored as there is little effect in films $\sim 100-500 \AA$ thick using $100 \mathrm{kV}$ electrons and in any case only intensity values are affected and not intensity peak positions.

The radial distribution function $R(r)$ and the pair distribution or correlation function $W(r)$ are obtained from the Fourier transform of $I_{\mathrm{m}}(s)$ i. e.

$$
\begin{aligned}
\ldots G(r)=4 & \pi r\left(\rho(r)-\rho_{0}\right)=\frac{2}{\pi} \times \\
& \times \int_{0}^{s_{\max }} s I_{\mathrm{m}}(s) \sin (r s) \exp \left(-B s^{2}\right) \mathrm{d} s
\end{aligned}
$$

as

$$
R(r)=4 \pi r^{2} \rho(r)=r G(r)+4 \pi r^{2} \rho_{0}
$$

and

$$
W(r)=\rho(r) / \rho_{0} .
$$

Here the symbols used have their usual meaning and $4 \pi r \rho_{0}$ is the initial slope of the reduced RDF $G(r)$. A damping factor and a finite limit to $s, s_{\max }$, are included in the integral to take advantage of the fact that $I_{\mathrm{m}}(s)$ decays rapidly with $s$ for electrons. In our results $s_{\max }=12.5 \AA^{-1}$ and $B$ is $0.015 \AA^{-2}$ so that $\exp \left(-B s_{\max }^{2}\right)=0.1$. The step length in the calculation was $\Delta s=0.018 \AA^{-1}$. In attempts to compare the experimental $I_{\mathrm{m}}(s)$ with that expected from small and deformed microcrystallites modified forms of the Debye equation were used [7].

4. Results of the structural analysis and resistance measurements. - An example of the scattering pattern from an amorphous cobalt film and its intensity trace, $I(s)$, are shown in figure 1 . The presence of a doublet in the second diffuse halo is quite evident. This pattern is typical in form of those obtained for all the films investigated i. e. cobalt; nickel, silver, gold and a Co-P alloy [7] and is also similar to X-ray scattering patterns obtained from amorphous metallic alloys $[2,4]$. The interference function extracted from $I(s)$ is shown in figure 2. The shape of the function and especially the strong-weak nature of the sub-peaks in the second halo appears to be typical of most solid amorphous films with metallic bonding. The errors introduced into $I_{\mathrm{m}}(s)$ using the extraction procedure from $I(s)$

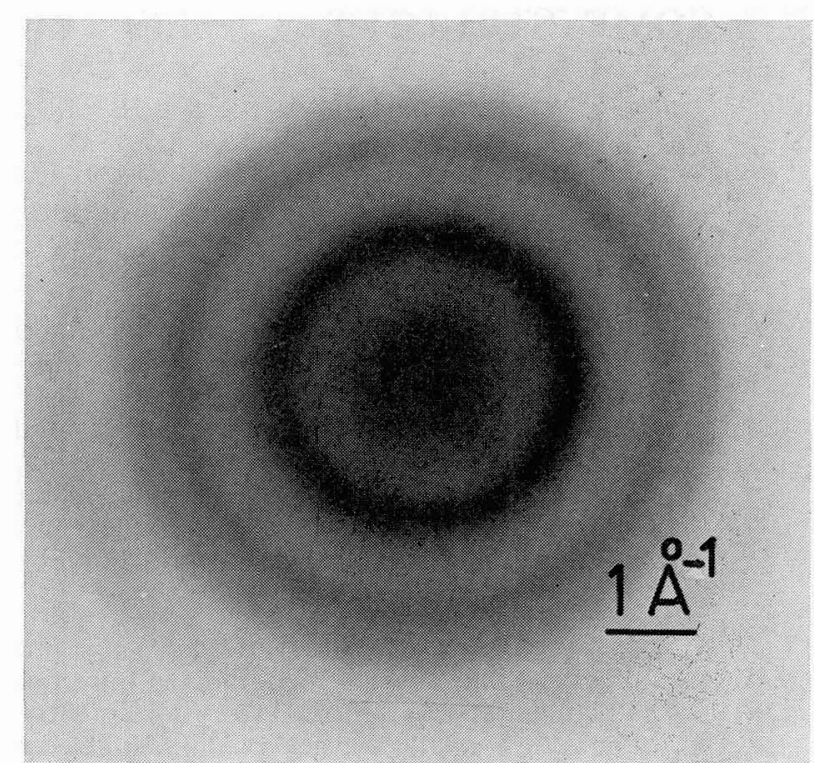

FIG. $1 a$.

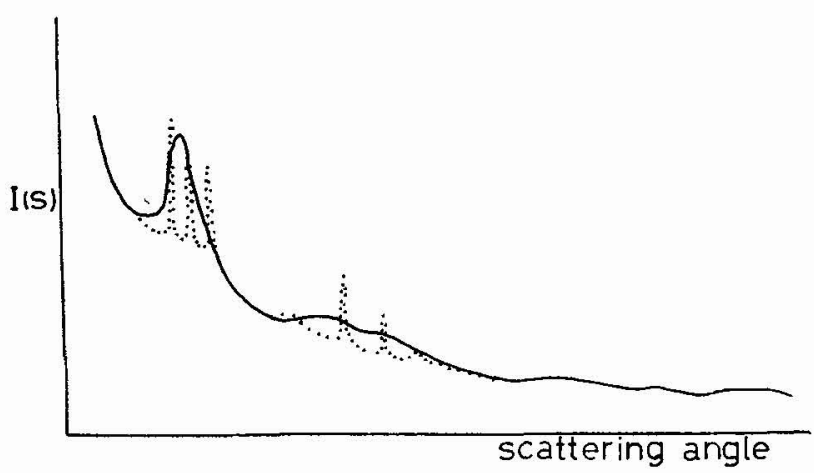

FIG. $1 b$.

Fig. 1. - Illustrating the electron scattering from a cobalt film vapour quenched at $77 \mathrm{~K}(a)$ the electron scattering pattern (b) a microdensitometer trace of (a) showing the total scattered intensity $I(s)$. The full curve is for the amorphous film and the dotted curve for the crystallized film.

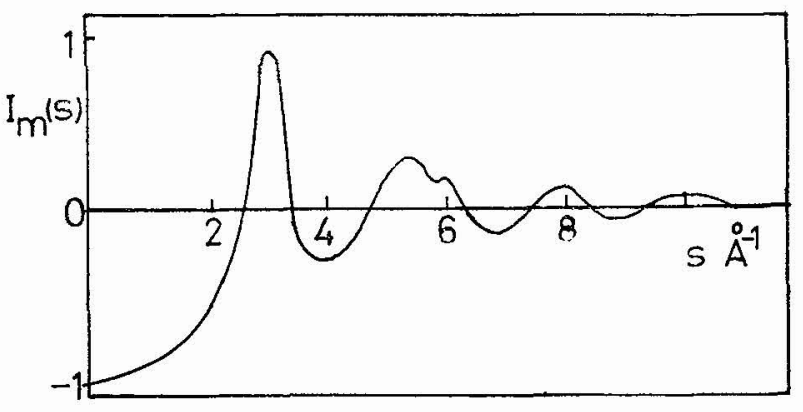

FIG. 2. - The interference function or structure sensitive scattering, $I_{\mathrm{m}}(s)$ extracted from figure $1 b . I_{\mathrm{m}}(s)$ is extrapolated to -1 at $s=0$ in ignoring low angle scattering. 
are unclear. Any contribution, especially at low angles, from scattering from specimen defects such as distributions of voids, etc. has been ignored. However the $I_{\mathrm{m}}(s)$ is reproducible from experiment to experiment certainly as far as the peak positions are concerned which are reproducible to about $0.01 \AA^{-1}$ in $s$. The ratio of these peak positions are very similar for all the specimens studied $s_{\mathrm{n}} / s_{1}=1.00: 1.70 ; 1.86: 2.49$ : 3.25 ; these are to be compared with $1.00: 1.69: 1.97$ : $2.54: 3.39$ for iron films [9].

An RDF $R(r)$ and a PCF $W(r)$ calculated from eq. (1) are shown in figure 3 . The curves for cobalt and nickel

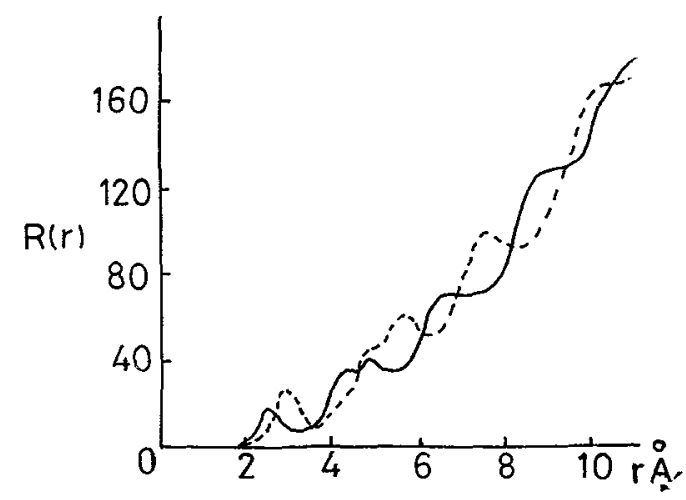

(a)

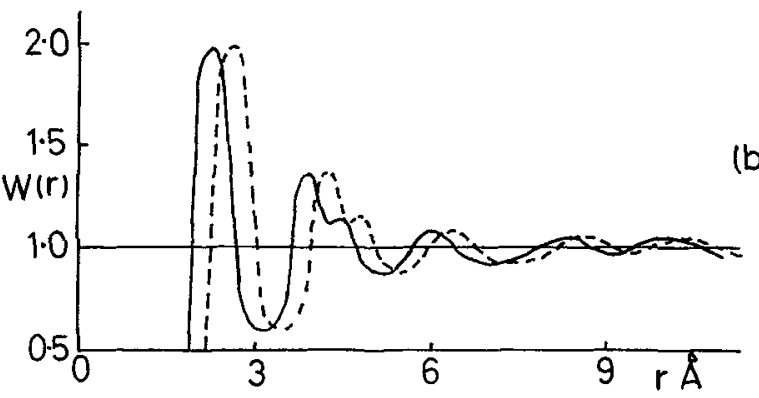

FIG. 3. - The radial distribution function $R(r)(a)$ and the pair correlation function $W(r)(b)$ for cobalt and nickel (solid curve) and gold and silver (dashed curve).

and gold and silver effectively coincide. $R(r)$ has a clearly defined first peak and subsequent peaks overlap each other. As is well known the area under the first peak can be used to estimate the first coordination number. For our films we obtain a value of $13 \pm 1$ which is to be compared with the close packed value of 12 in the crystalline phase. Because of the non-zero value of $R(r)$ at the first minimum not much wejght can be given to this value but it is in agreement with values obtained for amorphous Ni-P alloys [2]. $W(r)$ gives the most commonly held interatomic distances in the films and, along with the calculated $R(r)$, can be used to give the reduced atomic spacings which produce the experimentally obtained interference function. This procedure is discussed in the next section. The error in the peak positions in our $R(r)$ and $W(r)$ is estimated to be about $0.01 \AA$ and the ratio of the peak positions is also accurate to these limits.

As the temperature of the deposited films was increased from $77 \mathrm{~K}$ crystallization was signified by the appearance of lines in the scattering pattern recognisable as crystalline reflections. Crystallization could be arrested by keeping the film at constant temperature but the atomic ordering process was not reversible. The ordering process was followed by electrical resistance measurements [10] and a typical result for a cobalt film is shown in figure 4 . The anneal to room

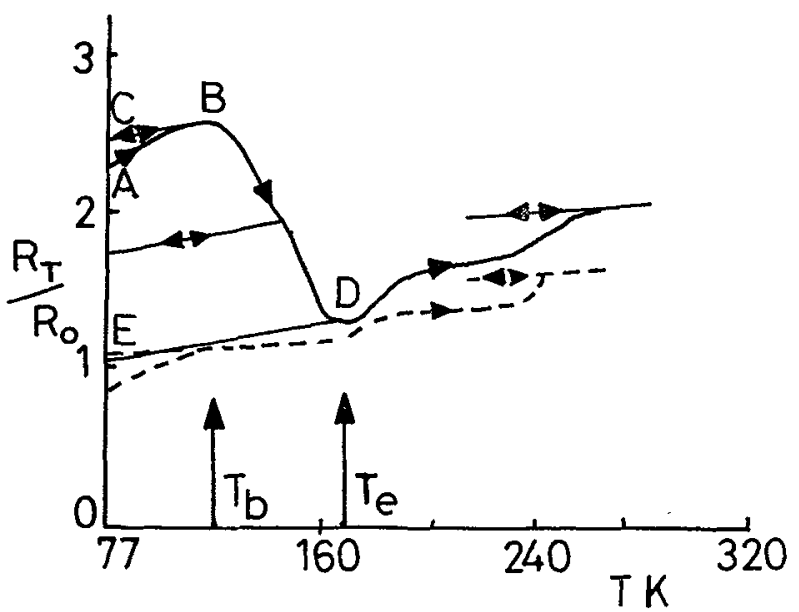

FIG. 4. - A plot of the reduced resistance of a vapour quenched cobalt film as a function of temperature. The dashed curve is for a crystalline iron film. $R_{0}$ at $0 \mathrm{~K}$ is obtained from $R_{\mathrm{e}}=R_{0}(1+\alpha T)$ where $\alpha$ is the temperature coefficient of resistance associated with DE.

temperature is characterised by three regions of behaviour. From $77 \mathrm{~K}$ to $T_{\mathrm{b}}$, i. e. A to $\mathrm{B}$, the disordered or amorphous phase is assumed to be stable, however the small rise in resistance is irreversible and the line BC is followed on cooling. Such an increase in resistance before crystallization has been ascribed to the formation of microcrystallites which increase the average scattering cross section [18]. Between B and D ordering or crystallization occurs with a marked drop in resistance and above $T_{\mathrm{e}}$ the film is crystalline with a positive temperature coefficient of resistance. The region over which crystallization occurs is irreversible and curves parallel to $\mathrm{DE}$ are followed on cycling the temperature. The behaviour of a crystalline film between $77 \mathrm{~K}$ and room temperature is entirely different as shown by the dotted curve. Resistance curves of this type have already been discussed in detail $[8,10]$ and analysed in terms of crystallization from a disordered, amorphous phase. Typical temperatures for

\section{TABLE I}

Temperatures at the beginning $\left(T_{b}\right)$ and end $\left(T_{\mathrm{e}}\right)$ of the crystallization in vapour quenched films. $R_{\mathrm{b}} / R_{\mathrm{e}}$ is the ratio of resistance at $T_{\mathrm{b}}$ and $T_{\mathrm{e}}$

$\begin{array}{lccc}\text { Film } & T_{\mathrm{b}} \mathrm{K} & T_{\mathrm{e}} \mathrm{K} & R_{\mathrm{b}} / R_{\mathrm{e}} \\ \text { Cobalt } & 120 & 166 & 2.0 \\ \text { Nickel } & 113 & 183 & 2.1 \\ \text { Gold } & 123 & 179 & 2.3 \\ \text { Silver } & 113 & 175 & 2.1\end{array}$


the onset and completion of crystallization in our films are listed in table I. This range of temperatures bears resemblance to those quoted by Ichikawa [9], but films deposited in UHV have a much lower and narrower range over which crystallization occurs as shown by Wright and his co-workers [8, 14]. This point is considered later.

5. Discussion of the scattering results. - Two procedures in analysing the scattering results have been followed in our work. The first is to calculate the scattering from microcrystalline structures and compare the computed interference functions with the experimental ones. Secondly the interference function and the atomic arrangement suggested by the experimental results can be compared with those obtained from model disordered structures such as the dense random packing of hard spheres (DRPHS) model of Finney [11] and the models simulating film deposition by Bennett [12], Sadoc et al. [13], Ichikawa [9] and Leung and Wright [14]. The first procedure has been described previously [7] and has also been followed by others, e. g. [9].

We have calculated the interference function for small f.c.c. and h.c.p. crystallites containing typically 9 to 81 atoms using the Debye equation and modifications to that equation. These modifications reflected the presence of stacking faults and elastic strains. The modifications essentially introduce nonuniform broadening into $I_{\mathrm{m}}(s)$ peaks, as is the case in the experimental $I_{\mathrm{m}}(s)$, whereas the simple reduction of crystallite size introduces uniform broadening. Ichikawa [9] considered small crystals of b. c. c., f. c. c., b. c. t., h. c. p. and A15 structure incorporating some disorder. The results of such calculations are, in the main, in complete disagreement with the experimental structure factor but in some cases the most important feature, the split nature of the second halo, is reproduced. However the relative intensities of the sub-peaks are usually in the wrong order i. e. weakstrong rather than strong-weak and where the correct order is obtained the peaks in $I_{\mathrm{m}}(s)$ and any $W(r)$ occur at the wrong values of $s$ and $r$.
Table II lists the ratio of the peaks in the pair correlation functions calculated by eq. (1) from experimental determinations of $I_{\mathrm{m}}(s)$ and from packing models. The preferred comparison is between the experimental $I_{\mathrm{m}}(s)$ and that calculated from the atomic coordinates in the model as done by Sadoc et al. [13] and Ichikawa [9]. The ratios of peaks in Sadoc's calculated $I_{\mathrm{m}}(s)$ for 1000 spheres in DRP are in good agreement with our results i. e. $s_{\mathrm{n}} / s_{1}=1.00: 1.70: 1.85$. A comparison between the calculated and experimental $I_{\mathrm{m}}(s)$ avoids any termination effects that may be introduced in calculating $R(r)$ or $W(r)$, however for a general overview a comparison of peak ratios in the $R(r)$ and $W(r)$ is convenient. The methods adopted in the setting up of the model atomic packings have been discussed fully by their originators. The procedures adopted in [9] and [12-14] are of particular interest because they are meant to be representative of the way in which deposited films nucleate and grow. The local and global models of Bennett are variants of a model where particles are deposited into hard contact with a triangular seed cluster. The global model simulates spherical packing and the local model a packing determined by short range forces. Sadoc et al chose a packing which contains no crystalline structure by building up a model with icosahedral symmetry from a similar seed. Ichikowa also used icosahedra but considered tetrahedral and dodecahedral seed clusters as well. The success of arrangements of perfect or distorted tetrahedra in predicting the experimental results is probably significant.

It can be seen from table II that the spherical DRP's of Finney and Bennett do not agree too well with the experimental results over the first few ratios, although it should be pointed out that Finney's model does predict a possible spacing of 1.65. The local models of Bennett and Sadoc et al. agree well with experiment at short range. All experimental values of $r_{3} / r_{1}$ are significantly less than 2.00 and, in genes al, experimental values at and beyond $r_{3} / r_{1}$ are in some disagreement with the models considered but the results of a recent model calculation by Wright [14] appear to be in better agreement. We include in the

\section{TABLE II}

Peak sin the pair correlation functions $W(r)$ from experimental interference functions and from structural models

\begin{tabular}{|c|c|c|c|c|c|c|}
\hline Film or model & $r_{1} / r_{1}$ & $r_{2} / r_{1}$ & $r_{3} / r_{1}$ & $r_{4} / r_{1}$ & $r_{5} / r_{1}$ & Ref. \\
\hline $\mathrm{Co}, \mathrm{Ni}, \mathrm{Au}, \mathrm{Ag}$ & 1.00 & $1.65_{2}$ & 1.90 & 2.55 & 3.46 & [7] \\
\hline Co & 1.00 & $1.69^{\circ}$ & 1.93 & 2.49 & 3.36 & [14] \\
\hline $\mathrm{Fe}$ & 1.00 & 1.67 & 1.96 & 2.51 & $3.38 \mid$ & \\
\hline $\mathrm{Ni}$ & 1.00 & 1.71 & 1.93 & 2.55 & $3.38\}$ & \\
\hline Co-P & 1.00 & $1.65_{5}$ & 1.90 & 2.55 & 3.45 & \\
\hline $\mathrm{Ni}-\mathrm{P}$ & 1.00 & 1.71 & 1.91 & & & {$[2$} \\
\hline Sadoc et al. & 1.00 & 1.65 & 2.00 & & & {$[1$} \\
\hline Local & 1.00 & 1.68 & 2.00 & & & \\
\hline Global $\}$ & 1.00 & 1.73 & 2.00 & 2.68 & 3.55 & \\
\hline Finney & 1.00 & 1.73 & 2.00 & 2.65 & 3.50 & L \\
\hline
\end{tabular}


Table our results for Co-P and those for a Ni-P alloy by Cargill [2] which, taken with the results for the vapour quenched films, illustrate an apparent general trend for atomic arrangements in amorphous metal and metallic alloy films. The actual values of $r_{1}$ for the amorphous phases are not significantly different (at the most $1-5 \%$ from the closest distance of approach in the crystalline phase, $i$. e. for nickel we have $r_{1}=2.50$ [7] and 2.48 [9] and for crystalline nickel $r_{1}=2.49 \AA$. The model shown in figure 5 representing two co-basal

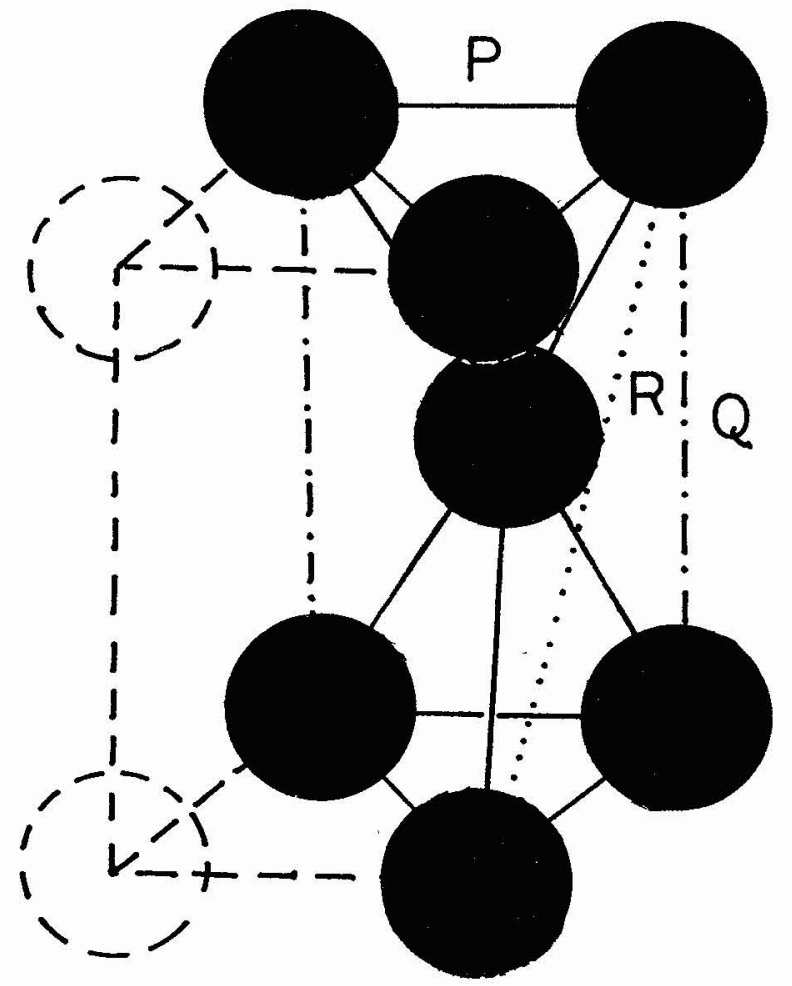

FIG. 5. - A cluster of two cobasal tetrahedra obtained from a deformed h.c. p. primitive cell giving spacings $p: q: r$ of $1: 1.63_{2}: 1.92$.

tetrahedra obtained from a distorted h. c. p. cell gives spacings in the ratio $1: 1.63_{2}: 1.91_{6}$ in good agreement with the experimental results. This kind of cluster is the correct size for growth of a DRPHS at longer range. The spherical DRPs of Finney and Bennett have a packing very similar to that in the close packed planes of a f. c. c. or h. c. p. system i. e. $1: 1.732: 2: 2.65$. It is thus clear that the experimental atomic arrangements are displaced further from the crystalline phase than suggested by a spherical DRPS and represent a distinct amorphous phase. Conditions of local order appear to extend to longer range than fixed by the local models considered here and models which are dense locally but relaxed at longer range maybe pertinent.

6. Magnetization measurements. - Of some interest at the present time is the general topic of amorphous magnetism and many reports have been published of magnetic measurements on non-crystalline ferro- magnetic [19] and ferrimagnetic films [3] (see also other papers in the conference proceedings of [1]). However little attention has apparently been paid to magnetization measurements in nominally pure ferromagnetic films apart from the investigation reported in [6]. Recently reports on amorphous transition metal alloys such as $\mathrm{NiFe}$ vapour quenched films have appeared [20] and magnetic measurements have been made on amorphous films of rare earth-transition metal alloys of importance in bubble domain technology [21].

Part of our programme is to investigate the magnetic properties of amorphous single element and alloy ferromagnetic films and some preliminary results on the saturation magnetization, $M_{\mathrm{s}}$, as a function of temperature for vapour quenched nickel and cobalt films are shown in figure 6 . These results were quite

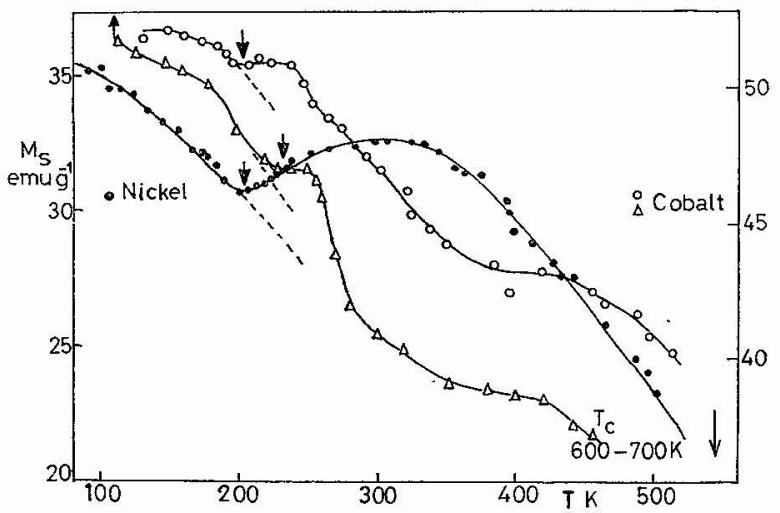

Frg. 6. - The magnetization of nickel and cobalt films vapour quenched at $77 \mathrm{~K}$ as a function of temperature.

reproducible in form and magnitude for several films deposited at the same pressure of $10^{-5}$ torr ; this is indicated by the curves for two separate cobalt films (both about $400 \AA$ thick). The curve for nickel is typical of films between about 500 and $2000 \AA$ in thickness. The results for nickel can be explained as follows. Below $200 \mathrm{~K}$ the film is assumed to be in a stabilised amorphous phase ; crystallization starts at this point initiated possibly by outgassing and the magnetization rises as ordering proceeds before falling along a normal magnetization curve to the accepted Curie point, $T_{\mathrm{c}}$, for the crystalline phase. It can be seen that if our assumptions are correct the $T_{\mathrm{c}}$ for the amorphous phase is higher than the crystallization temperature. This is in disagreement with Tamura and Endo's results [6] where, assuming the behaviour of their film was ferro- rather than ferrimagnetic, the amorphous $T_{\mathrm{c}}$ coincided with the crystallization temperature of the film. This temperature of $530 \mathrm{~K}$ is remarkably high for a film deposited at their quoted vacuum of $4 \times 10^{-7}$ torr. For our nickel films the ratio of the $M_{\mathrm{s}}$ in the two phases, extrapolated to $0 \mathrm{~K}$, are $M_{\mathrm{SA}}(0) /$ $M_{\mathrm{SC}}(0) \simeq 0.7$ and for $T_{\mathrm{c}}, T_{\mathrm{CA}} / T_{\mathrm{CC}} \simeq 0.6$.

The curves for cobalt appear to be a little more complex. It is reasonable to suppose from the struc- 
tural work and the results for nickel that some form of crystalline ordering occurs at about $200 \mathrm{~K}$. From that point the magnetization in the crystalline phase follows, initially, a magnetization curve reminiscent of that for a specimen composed of small ferromagnetic clusters identified with small grains $(\sim 10-50 \AA$ in size) which interact with each other through magnetostatic or rudimentary exchange interactions [22, 23]. It would appear that above about $350-400 \mathrm{~K}$ a normal magnetization curve begins. If this is the case it is presumably the result of substantial annealing by removal of gaseous impurities and some grain growth. If this explanation of the cobalt results is correct the difference in magnetization at any temperature between the amorphous and crystalline phase is small and much smaller than for nickel. This is in agreement with measurements on the Hall effect [15] and Mössbauer studies by Hoffman [14] for cobalt where the internal field for the amorphous phase $\sim 5 \%$ less than that for the crystalline phase.

A more extensive investigation is in progress on films condensed at liquid helium temperatures and in improved vacua.

7. Conclusion. - It is clear from the structural results considered in this paper that single element metallic films of the transition series and coinage metals can be prepared in an amorphous phase. It can be concluded that in most cases the presence of residual gases during film deposition serves to stabilise an amorphous phase at low temperatures by inclusion of gas molecules in the film. It would seem that only cobalt can be obtained in a pure, amorphous state [8] and iron incertain circumstances [14]. At pressures of $10^{-9}$ torr the impurity content of thin films is estimated to be $\sim 0.1 \%$. Nickel films deposited at any temperature in vacua better than $10^{-6}$ torr are always microcrystalline. Our films probably have an impurity content of about 1-2\% for fast depositions and are presumably stabilised in the amorphous phase by the gas molecules acting as a dilute glass former. Hence contrary to some suggestions single element metal films can be obtained in an amorphous phase and it would seem that metallic bonding can support a disordered structure. Covalent binding as occurs in semiconductors is not the only binding mechanism to support an amorphous state. Part of the discussion of sections 4 and 5 has recently been reported [24].

The magnetic measurements reported in this paper are only preliminary; they and others will be discussed more fully elsewhere [16]. However it is clear that interesting information will be forthcoming on amorphous ferromagnetic material of the most fundamental kind-pure metals. We hope to effect some kind of comparison with theoretical treatments of this state.

The atomic arrangement in amorphous films of the metals considered here appear to be explained, at least in principle, by recently proposed models of dense random packing of hard spheres built up by sequentially adding new spheres to a particular seed cluster under certain conditions of packing. Where packing is governed by local considerations, i. e. short range forces, the calculations and experiment are in good agreement at short range. Successful models seem to be those where addition of an atom in hard contact to three others already in hard contact to make a seed in the form of a tetrahedron is a distinctive feature.

\section{References}

[1] Tsuer, C. C., Amorphous Magnetism, ed. H. O. Hooper and A. M. de Graaf (Plenum Press) 1973 p. 299.

[2] Cargill III, G. S., J. Appl. Phys. 41 (1970) 12.

[3] Simpson, A. W. and Brambley, D. R., Phys. Stat. Sol. (b) 49 (1972) 685.

[4] Dixmier, J. and Duwez, P., J. Appl. Phys. 44 (1973) 1189.

[5] Fujrme, S., Japan J. Appl. Phys. 6 (1967) 305 and 5 (1966) $1029,778$.

[6] Tamura, K. and Endo, H., Phys. Lett. 29A (1969) 52.

[7] Davies, L. B. and Grundy, P. J., Phys. Stat. Sol. (a) 8 (1971) 189.

[8] Bennett, M. R. and Wright, J. G., Phys. Stat. Sol. (a) 13 (1972) 135.

[9] Ickikawa, T., Phys. Stat. Sol. (a) 19 (1973) 707.

[10] Davies, L. B. and Grundy, P. J., J. Non-Cryst. Solids 11 (1972) 179.

[11] FinNey, J. L., Proc. R. Soc. Lond. A 319 (1970) 479.

[12] BenNetT, C. H., J. Appl. Phys. 43 (1972) 2727.

[13] SADOC, J. F., DIXMIER, J. and GuINIER, A., J. Non-Cryst. Solids 12 (1973) 46
[14] Leung, P. K. and Wright, J. G., to be published in Phil. Mag. and private communication (1974).

[15] Whyman, P. and Aldridge, R. V., J. Phys. F 4 (1974) L6.

[16] NandRa, S. S. and GRUNDY, P. J., to be published.

[17] ICHIKAWA, T., to be published.

[18] Chen, H. S. and Turnbull, D., J. Chem. Phys. 48 (1968) 2560.

[19] Cochrane, R. W. and Cargill III, G. S., Phys. Rev. Lett. 32 (1974) 476.

[20] Nose, H., Proc. Int. Conf. on Magnetism, Moscow, paper 24M-S3 (1973).

[21] TaO, L. J., Gambino, R. J., Kirkpatrick, S., Cuomo, J. J. and Lilienthal, H., AIP Conf. Proc. on 19th Magnetism and Magnetic Materials Conf., paper 8E-3 (1973).

[22] Bostanjoglo, O. and Röhkel, K., Phys. Stat. Sol. (a) 7 (1971) 387.

[23] Cusick, J. P., Bambakidis, G. and Becker, L. C., Amorphous Magnetism, ed. H. O. Hooper and A. M. de Graaf (Plenum Press) 1973 p. 291.

[24] Grundy, P. J. and Davies, L. B., J. Phys. F 4 (1974) LIII. 